Proc. 19th Winter Workshop on

Nuclear Dynamics (2003) 000-000

\begin{tabular}{l}
\hline 19th Winter Workshop \\
on Nuclear Dynamics \\
Breckenridge, Colorado, USA \\
February 8-15, 2003 \\
\hline \hline
\end{tabular}

\title{
Strangeness Saturation: Dependence on System-Size, Centrality and Energy ${ }^{a}$
}

\author{
B. Kämpfer ${ }^{1}$, J. Cleymans ${ }^{2}$, P. Steinberg ${ }^{2, b}$ and S. Wheaton ${ }^{2}$ \\ ${ }^{1}$ Forschungszentrum Rossendorf, PF 510119, 01314 Dresden, Germany \\ 2 Department of Physics, University of Cape Town, \\ Rondebosch 7701, Cape Town, South Africa
}

Abstract. The dependence of the strangeness saturation factor on the system size, centrality and energy is studied in relativistic heavy-ion collisions.

Keywords: relativistic heavy-ion collisions, statistical hadronization PACS: 24.10.Pa, 25.75.Dw, 25.75.-q, 27.75.+p, 12.38.Mh, 24.85.+p

\section{Introduction}

Statistical-thermal models (see [1] for a review) aim at reproducing hadron multiplicities in relativistic heavy-ion collisions in certain phase space regions by a small number of parameters. As these parameters evolve smoothly with external conditions, thermal models have predictive power. Moreover, the thermal parameters, taken literally, allow for a quantification of the conditions reached in the transient stage of compressed and heated strongly interacting matter. While there is exhaustive literature on the successful use of the thermal models, the foundation of the applicability is far from settled. One should also keep in mind that thermal models address only a subset of the wealth of observables.

The present contribution summarizes our recent work [ 2, 3, 4] investigating the dependence of strangeness saturation on system size, centrality and energy, both in full phase space $(4 \pi)$ and at mid-rapidity $(y \approx 0)$.

\section{Thermal Model}

Our model is based on the grand canonical ensemble expression for the abundance of hadron species $i$ (Fermions $[+1]$ or Bosons [-1]) with degeneracy $g_{i}$

$$
N_{i}^{\text {prim }}=V g_{i} \int \frac{d^{3} p}{(2 \pi)^{3}} d m_{i}\left[\gamma_{s}^{-\left|S_{i}\right|} \exp \left\{-\frac{E_{i}-\vec{\mu}_{i} \vec{Q}_{i}}{T}\right\} \pm 1\right]^{-1} \operatorname{BW}\left(m_{i}, \Gamma_{i}\right),
$$


where $T$ is the temperature, $\vec{\mu}$ a set of chemical potentials corresponding to the conserved charges $\vec{Q} ; E_{i}=\sqrt{\vec{p}^{2}+m_{i}^{2}}$ with vacuum mass $m_{i}$.

For small particle numbers one has to turn to a canonical ensemble, e.g. by the projection method [ 5]. It is important to include resonances (we include meson (baryon) states composed of the three lightest quarks and anti-quarks up to $2.3(2.6) \mathrm{GeV}$ ); their widths are taken into account via the Breit-Wigner parameterization $B W\left(m_{i}, \Gamma_{i}\right)$, which collapses to a $\delta$-function for the respective ground states. Due to feeding and resonance decays, the final hadron multiplicities become $\left.N_{i}=N_{i}^{\text {prim }}+\sum_{j}\left\{N_{j}^{\text {prim }} B r(j \rightarrow i)-N_{i}^{\text {prim }} B r(i \rightarrow j)\right]\right\}$, where $B r$ denotes the corresponding branching ratio.

Our focus here is the phenomenological strangeness saturation factor $\gamma_{s}$ in Eq. (1). It is thought [ [6] to parameterize possible deviations from chemical equilibrium in the strange sector. Whether one common factor $\gamma_{s}$ is sufficient, with powers $\left|S_{i}\right|$ determined by the total strangeness content of hadron $i$, needs still to be tested by analyzing data. Also in the non-strange sector such an off-equilibrium parameter should be introduced [ [7]. Due to the small data samples at our disposal, such an additional parameter cannot be unambiguously fixed; therefore, we are forced here to assume full equilibration in the non-strange sector.

The parameters $T$ and $\vec{\mu}$ appear exponentially in Eq. (1), while the sensitivity on variations of $\gamma_{s}$ is weaker. For this reason, we study the mentioned parameter dependencies based on analyses of data sets comprised of the same hadron species. Otherwise variations of $\gamma_{s}$ can be absorbed in small changes of $T$ and $\vec{\mu}$. Unfortunately, this shrinks the wealth of available data to quite poor subsets.

Within the Cooper-Frye formalism, dynamical effects factor out of fully-integrated hadron yields [ [8], provided that freeze-out occurs on a hyper-surface with constant $T$ and $\vec{\mu}$. In this respect, Eq. (1) covers also dynamical situations. Instead of considering hadron ratios, we consider multiplicities $N_{i}^{\text {prim }}$ with a fiducial normalization volume $V$ in Eq. (1).

\section{Data Analysis}

\section{1. $C E R N-S P S$ energies}

\subsubsection{Centrality and System Size Dependence at $E_{\text {beam }}=158 \mathrm{AGeV}$}

$4 \pi$ multiplicities of $\pi^{ \pm}, K^{ \pm}, \phi$ and $N_{\text {part }}$ are at our disposal for (i) central collisions of $\mathrm{C}+\mathrm{C}$ and $\mathrm{Si}+\mathrm{Si}$, and (ii) centrality binned collisions $\mathrm{Pb}+\mathrm{Pb}$. More baryon information is desirable, but only $\bar{p}$ 's are available additionaly for $\mathrm{Pb}+\mathrm{Pb}$. The left panel of Fig. 1 summarizes our findings [3]. We observe,

- $\mathrm{Pb}+\mathrm{Pb}: \gamma_{s}$ increases with centrality, but stays below unity, irrespective of whether $\vec{p}$ 's are included or not; inclusion of $\bar{p}$ 's reduces $\gamma_{s}$ somewhat; minimizing $\chi^{2}$ or the quadratic deviation of data and the model multiplicities, $q^{2}$, yields slightly different results.

- $\mathrm{C}+\mathrm{C}, \mathrm{Si}+\mathrm{Si}$ : the extracted values of $\gamma_{s}$ off-set the down-extrapolation for $\mathrm{Pb}$ 
$+\mathrm{Pb}$. This again emphasizes the findings of NA49 [9]: system-size and centrality dependencies differ.

- Most striking is that $\gamma_{s}$ goes as the number of participants which underwent multiple collisions, as extracted from a Glauber model calculation (cf. Fig. 3 in [ 3]).

There is some controversy on the use of $4 \pi$ or mid-rapidity data. Our analysis [ 3] of $\pi^{ \pm}, K^{ \pm}$and $p^{ \pm}$centrality-binned mid-rapidity data from $\mathrm{Pb}+\mathrm{Pb}$ at $158 \mathrm{AGeV}$ reveals that $\gamma_{s}$ does indeed differ for $4 \pi$ and $y \approx 0$ data (see Fig. 1, middle panel). This is due to the fact that the rapidity distributions of various hadrons differ. Combining the results, even the very restricted data samples point to $\gamma_{s}=1 \pm 0.25$ for most central collisions. This suggest that $\gamma_{s}=1$ with slightly renormalized values of $T$ and $\vec{\mu}$ is a good choice for the given beam energy and high centrality.

\subsubsection{Energy Dependence}

Unfortunately, the previous analysis cannot be extended to extract energy dependencies. Rather, the $4 \pi$ multiplicities of $\pi^{ \pm}, K^{ \pm}, \Lambda$ and $\bar{\Lambda}$ at beam energies of 40, 80 and $158 \mathrm{AGeV}$ are at our disposal as comparable data samples. The results of our analysis [3] are shown in Fig. 2: $\gamma_{s}$ displays a dip at $80 \mathrm{AGeV}$, while $\gamma_{s}(40 \mathrm{AGeV}) \sim \gamma_{s}(158 \mathrm{AGeV})$. (The significance of the drop at $80 \mathrm{AGeV}$ still needs to be clarified.) $\gamma_{s}(158 \mathrm{AGeV})$ is compatible with the strangeness saturation factor reported in the previous subsection emerging from a data set suitable for a centrality analysis. The open circle follows from an analysis of mid-rapidity data of $\pi^{ \pm}$, $K^{ \pm}, \Lambda$ and $\bar{\Lambda}$. The change relative to results reported in the previous subsection highlights the sensitivity on different data sets, if the number of analyzed hadron species is small.

\subsection{RHIC Energies}

Our analysis is restricted to the published data of $\pi^{ \pm}, K^{ \pm}$and $p^{ \pm}$at $y \approx 0$ in centrality-binned collisions of $\mathrm{Au}+\mathrm{Au}$ at $\sqrt{s_{N N}}=130 \mathrm{GeV}$ [10]. The upcoming data for further hadron species, including excited states, and data at $\sqrt{s_{N N}}=200$ $\mathrm{GeV}$ will complete our picture on the systematics of hadron multiplicities.

\subsubsection{Centrality Dependence}

As for CERN-SPS energies, $\gamma_{s}$ increases with centrality, see right panel in Fig. 1. The overall trend follows again the number of multiple participant collisions obtained in a Glauber model calculation [ 3]. The most central collisions point to strangeness saturation, in particular when feed-down from weak decays is included. 


\subsubsection{Energy Dependence}

Fig. 2 suggests that increasing energy causes increased strangeness saturation. However, this conclusion should be taken with caution, as Fig. 2 displays in one plot analyses of $4 \pi$ and $y \approx 0$ data and data sets comprising different hadron species. Assuming that at CERN-SPS the $4 \pi$ data are sensible, while at RHIC only $y \approx 0$ data should be analyzed within thermal models, then from Fig. 2 one does indeed derive the mentioned expectation: with increasing energy, $\gamma_{s}$ evolves towards saturation.

One observes in Fig. 2 that the thermal model delivers for $p p$ and $\bar{p} p$ collisions [11] values of $\gamma_{s}$ compatible with peripheral collisions.
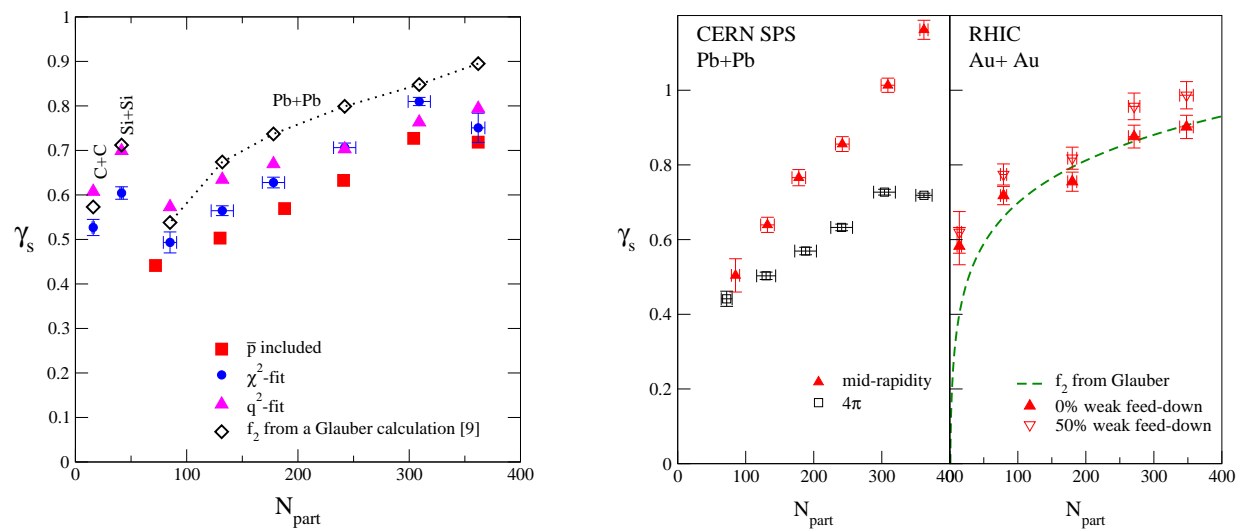

Fig. 1. Left panel: System-size and centrality dependencies of $\gamma_{s}$, as extracted from centrality-binned $\mathrm{Pb}+\mathrm{Pb}$ [12, 13] and central $\mathrm{C}+\mathrm{C}$ and $\mathrm{Si}+\mathrm{Si}$ data [9] under various fit conditions, assuming $50 \%$ feeding from weak decays. Also shown is the fraction of participants which underwent multiple collisions, $f_{2}$. Middle panel: Comparison of $\gamma_{s}$ extracted from mid-rapidity NA49 data [12] with the results of

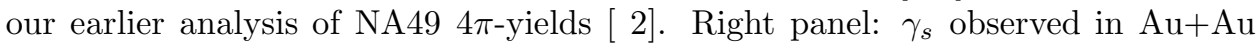
collisions as extracted from PHENIX data [ 10]; $f_{2}$ from our Monte Carlo based Glauber model calculation [3].

\section{Agreement with Data}

Up to now we have reported the system-size, centrality and energy dependence of $\gamma_{s}$. The values of $T$ coincide approximately with the chiral (deconfinement) transition temperature of $T \sim 165 \mathrm{MeV}$, smoothly increasing from $150 \mathrm{MeV}$ at beam energy of $40 \mathrm{AGeV} . \mu_{B}$ (the baryon potential dominating $\vec{\mu}$ ) decreases from 350 to $220 \mathrm{MeV}$ at CERN-SPS and drops to about $30 \mathrm{MeV}$ at RHIC. The actual values depend on the data samples analyzed. The agreement of data with multiplicities from the thermal model is extremely good with the following exceptions:

- $\phi / K^{+}$is underpredicted for peripheral collisions at beam energy of $158 \mathrm{AGeV}$, even if the $\phi$ yields are included in the fit; 


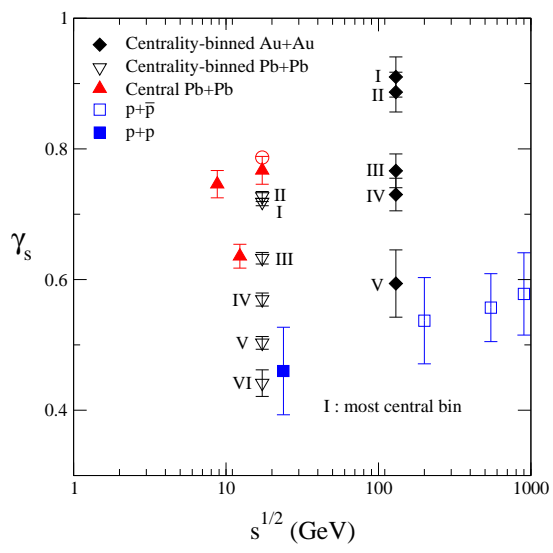

Fig. 2. Energy dependence of $\gamma_{s}$ extracted from central $\mathrm{Pb}+\mathrm{Pb}$ collisions at 40,80 and $158 \mathrm{AGeV}$ [14, 15, 16, together with the results of our earlier analysis of centrality-binned $\mathrm{Pb}+\mathrm{Pb}$ collisions at $158 \mathrm{AGeV}$ and $\mathrm{Au}+\mathrm{Au}$ collisions at RHIC [2]. The open circle is extracted from mid-rapidity yields [ 14, 16, 17]. Results for $p p$ and $p \bar{p}$ collisions from [11].

- $\Lambda+\Xi^{0}$ yields follow the data trend but are in absolute normalization below data when using the thermal parameters extracted from $\pi^{ \pm}, K^{ \pm}$and $p^{ \pm}$yields at $\sqrt{s_{N N}}=130 \mathrm{GeV}$; the $\Xi^{-}$yields are at the lower end of the error bars.

To understand which trends in the data drive the change of $\gamma_{s}$ we exhibit in Fig. 3 scaled multiplicities as a function of $N_{\text {part }}$. A scaling of $\bar{p}$ by $N_{\text {part }}^{1.15}$ results in a flat distribution at beam energy of $158 \mathrm{AGeV}$. The scaled $\pi^{ \pm}$is slightly decreasing, while $K^{ \pm}$increases. It is the relative decrease of the "gap" between pion and kaon yields which drives $\gamma_{s}$ towards unity. Similarly, at $\sqrt{s_{N N}}=130 \mathrm{GeV}$, the $p^{ \pm}$ distributions are flat, as also $K^{ \pm}$, but the $\pi^{ \pm}$decrease with increasing $N_{\text {part }}$. Again, the decreasing "gap" between $\pi^{ \pm}$and $K^{ \pm}$drives the increase of $\gamma_{s}$. Note that the effects are small and hardly visible in a log plot with unscaled yields.

As an aside, we mention that we adjusted the parameters of the color glass model [ 18] and obtain a good description of $\pi^{ \pm}$and $p^{ \pm}$yields, while the model deviates in details from the $K^{ \pm}$data, see right panel of Fig. 3.

\section{Summary}

In summary we report here the dependence of the strangeness saturation factor, $\gamma_{s}$, on system size, centrality and energy. Most striking is the increase of $\gamma_{s}$ with centrality and the number of multiple participant collisions. Details of the energy dependence of $\gamma_{s}$ are puzzling and hampered by restricted data sets. Whenever possible, one should compare the findings with results obtained from the richer data samples for central collisions. Clearly, the present investigations should be extended to include multi-strange baryons and resonances in the analysis of more complete data sets. This will further test the applicability and predictive power of thermal models of statistical hadronization in describing hadron multiplicities. 
B. Kämpfer et al.
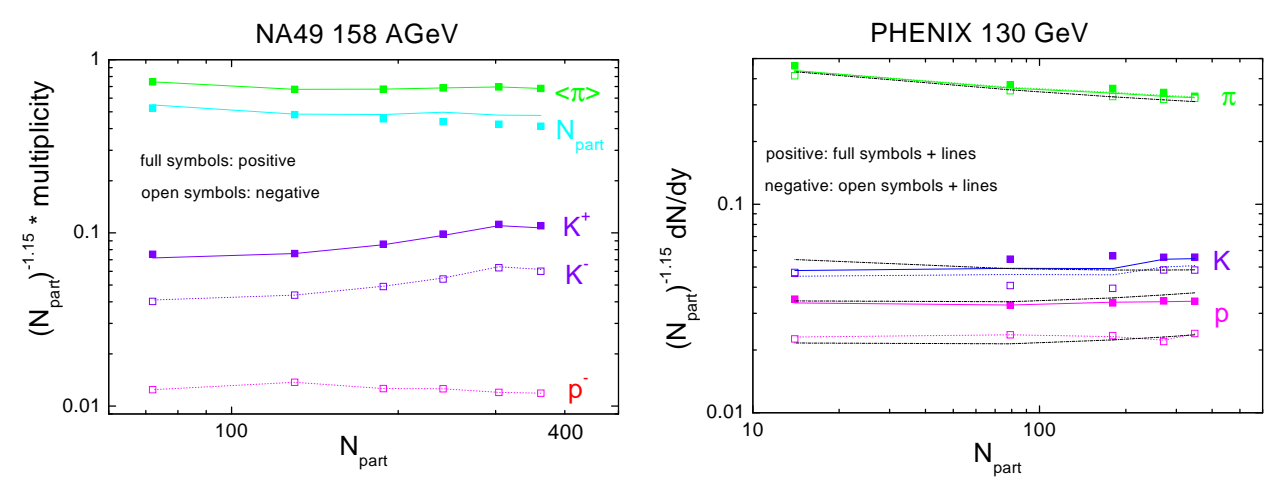

Fig. 3. Scaled hadron yields as a function of $N_{\text {part }}$. Left (right) panel: $\mathrm{Pb}+$ $\mathrm{Pb}(\mathrm{Au}+\mathrm{Au})$, symbols: data [12, 13, 10, dotted and solid lines: results of the thermal model, dot-dashed curves: our results for the color glass model [18].

\section{$\operatorname{Note}(\mathbf{s})$}

a. The work is supported by BMBF 06DR921 and 06DR121.

b. Visiting Fulbright Professor on leave of absence from the Brookhaven National Laboratory, Upton, NY, USA

\section{References}

1. P. Braun-Munzinger, K. Redlich and J. Stachel, nucl-th/0304013

2. J. Cleymans, B. Kämpfer and S. Wheaton, Phys. Rev. C65 (2002) 027901, Nucl. Phys. A 715 (2003) 553c.

3. J. Cleymans, B. Kämpfer, P. Steinberg and S. Wheaton, hep-ph/0212335

4. B. Kämpfer, J. Cleymans, K. Gallmeister and S. Wheaton, hep-ph/0202134. hep-ph/0204227

5. S. Hamieh, K. Redlich and A. Tounsi, Phys. Lett. B486 (2000) 61.

6. J. Letessier, J. Rafelski and A. Tounsi, Phys. Rev. C50 (1994) 406;

C. Slotta, J. Sollfrank and U. Heinz, AIP Conf. Proc. 340 (Woodbury, 1995), p. 462, (Ed.) J. Rafelski.

7. J. Rafelski and J. Letessier, nul-th/0209084

8. J. Cleymans and K. Redlich, Phys. Rev. C60 (1999) 054908.

9. C. Höhne (NA49 collaboration), nucl-ex/0209018

10. K. Adcox et al. (PHENIX collaboration), Phys. Rev. Lett. 88 (2002) 242301.

11. F. Becattini and U. Heinz, Z. Phys. C76 (1997) 269.

12. F. Sikler (NA49 collaboration), Nucl. Phys. A661 (1999) 45c.

13. V. Friese et al. (NA49 collaboration), Nucl. Phys. A698 (2002) 487c.

14. S.V. Afanasiev et al. (NA49 Collaboration), Phys. Rev. C66 (2002) 054902.

15. S.V. Afanasiev et al. (NA49 Collaboration), Phys. Lett. B491 (2000) 59. 
16. S.V. Afanasiev et al. (NA49 Collaboration), Phys. Lett. B538 (2002) 275.

17. A. Mischke (NA49 Collaboration), nucl-ex/0209002

18. L. McLerran and J. Schaffner-Bielich, Phys. Lett. B514 (2001) 29; J. Schaffner-Bielich, D. Kharzeev, L. McLerran and R. Venugopalan, Nucl. Phys. A705 (2002) 494. 